\title{
SOBRE EL SENTIDO DEL UNIVERSO Y LA EXISTENCIA
}

\author{
Miguel Ángel Ortega Sánchez. Madrid
}

\begin{abstract}
La pregunta por el sentido de la vida suele ser considerada como la pregunta más profunda de la filosofía, mundana o académica: incluso se llega a definir al hombre como «el ser capaz de interrogar por el sentido del ser», de su existencia, de su vida ...

Gustavo Bueno $\left({ }^{1}\right)$
\end{abstract}

Resumen: En el presente trabajo se justifica la extensión de los «principios de mínimo» de aplicación general en la física, tanto en la mecánica clásica como en la electrodinámica cuántica, a las ciencias sociales, en particular a la economía que se basa en criterios de óptimo (Óptimo Paretiano) y al ámbito social y político a través de la idea de Institución. Instituciones sometidas a criterios de eficiencia por medio de procesos de selección darwinista.

Palabras clave: Universo, Sentido del Universo, Principios de Mínima Acción, Principio Antrópico, Óptimo Paretiano, Institucionalismo, Instituciones sociales, Evolucionismo.

On the Sense of Universe and Existence

Abstract: This document provides justification for extending the «de minimis principles» that are widely applied in the field of physics (both in classical mechanics and in quantum electrodynamics) to the social sciences, particularly to economics based on optimum criteria (Pareto Optimum) and to the social and political environment through the concept of the Institution: institutions subjected to efficiency criteria through Darwinistic selection processes.

Keywords: Universe, Sense of Universe, "De minimis action principles", Anthropic Principle, Pareto Optimum, Institutionalism, Social Institutions, Evolutionism.

Recibido: 24/06/2018. Aprobado: 21/12/2018.

\section{Introduccion}

En el campo del conocimiento siempre ha existido o se ha tratado de establecer una clara división entre ciencias físicas y naturales y

1. Bueno, G. "El sentido de la vida", en Pentalfa, Oviedo 1996.

Naturaleza y Libertad. Número 11, 2019. ISSN: 2254-9668 
ciencias sociales. La naturaleza del objeto de estudio de cada uno de estos ámbitos: los objetos materiales inertes y la fisiología de los seres vivos por una parte y la actividad social de los seres humanos, resultado de su elección libre y consciente por otra, hace difícil establecer puentes entre los mismos. Los trabajos de Wilhelm Dilthey abundan en esta línea de diferenciación. Sin embargo, es claro que los seres humanos, por su naturaleza son elementos materiales del medio físico, existen en un entorno y dependen del medio natural con el que interaccionan. Pese a la importancia del tema se puede decir que hay pocos trabajos que tratan de conectar ambos ámbitos y cuando lo hacen, los puntos de vista son particulares $\left[\left({ }^{1}\right),\left({ }^{2}\right),\left({ }^{3}\right),\left({ }^{4}\right)\right]$. Pero más difícil es encontrar análisis generales en los que se formulen teorías que tiendan a buscar su unificación estableciendo principios comunes y al mismo tiempo traten de encontrar un sentido unitario de una realidad también única pero que aparentemente se muestra tan distinta. En esta línea destacan los trabajos de Karl Popper, aunque su planteamiento es metodológico. No obstante, como señala Kant, el científico no se contenta con acumular leyes sino que intenta descubrir interrelaciones entre ellas con el propósito de construir sistemas. Para ello, su investigación tiene que presuponer la unidad de la naturaleza como si fuera un sistema lógico.

Un ejemplo de esfuerzo en esta línea es la conferencia «Una Teoría del Todo. Patrimonio de la Escuela Austriaca de Economía» pronunciada por Eladio García García en el Instituto Juan de Mariana el 22VII-2015 y que se puede reproducir en YouTube.

En la presente exposición se trata de ofrecer una visión general e intuitiva de lo que se podría denominar «Teoría del Todo» (más allá de la física) con el sentido de englobar los aspectos físicos, sociales y humanos en los que se desenvuelve la existencia humana. Una descripción general, en la que se hayan considerado los elementos más

2. Méndez Molinero, R. "Espacios y sociedades. Introducción a la geografía regional del mundo", en Editorial Ariel Geografía. 1984.

3. Schrödinger, R. E. "Qué es la vida", en Textos de Biofísica. Facultad de Farmacia. Universidad de Salamanca, 2005.

4. Pombo, O.; Torres, J. M.; Symons, J.; Rahman, S. "Special Sciences and the Unity of Science", Springer Science. 2012.

5. van Bendegem, J. P.; Rahman, S.; Symon, J. "Logic, Epistemology, and the Unity of Science". Springer Science, 2009. 
importantes que caracterizan la realidad, debería proporcionar conclusiones sobre su naturaleza, su sentido y sus principios. Lo cual permitiría ordenar de forma lógica, con un sentido unitario los elementos de la misma.

Nuestra existencia muestra que la naturaleza ha evolucionada desde el mundo físico de la materia inerte, pasando por las transformaciones producidas por las reacciones de sustancias químicamente activas hasta el mundo de los seres vivos del reino vegetal y animal y finalmente el mundo social creado por la racionalidad humana gobernada por principios éticos o morales.

Estas realidades tan diferentes cada una de ellas muestran una gran complejidad. Tratar de explicar por qué y cómo suceden los acontecimientos para pronosticar el futuro ha sido objeto de la actividad científica que ha procurado analizar pormenorizadamente los distintos aspectos de la realidad. Pero al mismo tiempo se plantea la cuestión de si toda la diversidad y variedad no obedece a unos principios básicos comunes; si es posible encontrar un sentido unitario de la misma, una entidad básica sobre la que se organiza la realidad, o una causa última o primera que la explica, desde el mundo físico al social.

Una primera cuestión es la delimitación de los aspectos de la realidad con los que formular ideas unitarias. Un ejemplo de esto sería la descripción de la evolución de la realidad en términos de entropía que ha permitido llegar a establecer el segundo principio de la Termodinámica cuyo resultado nos muestra la evolución como desorden. Para llegar a este planteamiento fue necesario el avance de la ciencia en aspectos muy concretos, con un análisis minucioso y detallado del que finalmente surgieron principios de carácter general. En este sentido el desarrollo científico es la base para encontrar nuevos principios que expliquen el sentido de la realidad.

Pero más allá de los principios físicos, cuyo contenido, los propios instintos nos dicen que tenemos que seguir, aparecen consideraciones como las cuestiones estéticas y éticas, así como sobre la justicia o los juicios morales.

El área del pensamiento más inclinada a tratar de establecer principios generales y si es posible únicos y sencillos ha sido la filosofía. 
Miguel Ángel Ortega Sánchez

Los filósofos han tratado de contemplar a la luz de la razón, la realidad de una forma más amplia y general posible.

En esta posición de generalidad y unicidad del pensamiento destaca, por primera vez en el pensamiento moderno, Descartes. Para Descartes la íntima unidad de las ciencias no es sino la unidad y la coherencia que reclama la verdad allí donde se presenta, la que se expresa en la famosa metáfora de árbol de la sabiduría que recogió en su obra «Los Principios de la Filosofía»: la unidad del saber era anterior a su despliegue en diferentes disciplinas (posición esta última defendida por Aristóteles) y que descansaba en la propia unidad de la razón humana.

\section{El universo fisico y natural}

En la evolución del universo desde su inicio (la física actual está de acuerdo en que existió un instante inicial), hasta el momento presente, se pueden considerar cinco fases:

El universo inflacionario en el que durante un breve lapso de tiempo (10-32 segundos) el espacio crece rápidamente (Big Bang) apareciendo las partículas elementales formando el Plasma Quark-Gluón con fotones, todos ellos en constantes choques, denominado también universo de Planck. Alǵunos autores sugieren que la inflación tiene lugar en un campo cuántico fluctuante preexistente en el que localmente se produce una gran fluctuación, marcando el tiempo $\mathrm{t}=0$ con el que se inicia el universo, pero es solo a partirdel tiempo 10-42 segundos, cuando la física es capaz de explicar la evolución posterior mediante el denominado «modelo estándar» (una descripción muy conocida es la de Steven Weinberg $\left({ }^{5}\right)$ ). Más adelante se hacen unas conjeturas sobre estos 10-42 segundos inalcanzables.

El universo opaco de formación de los elementos básicos: las partículas elementales acaban organizándose, aproximadamente, en 3/4 partes de Hidrogeno, $1 / 4$ de Helio y pequeñas porciones de litio y berilio al cabo de 380000 años. Momento en el que los fotones de luz pudieron viajar con cierta libertad dejando detrás de sí la radiación de fondo

6. Weinberg, S. "Los tres primeros minutos del Universo", en Alianza Editorial, 1980. 
que en la actualidad muestra la distribución de materia y energía no uniforme que existió en aquel momento.

El universo oscuro durante el cual la materia existente va formando las grandes estructuras del universo que tienen su origen en la falta de isotropía que caracterizó el fin de la fase anterior y que dieron lugar a las galaxias, nebulosas, ete. Estructuras en las que al cabo de $400 \mathrm{Mi}$ llones de años aparecen las primeras estrellas iluminando de forma tenue el universo.

Formación de sistemas planetarios en torno a las estrellas y en aquellos que se dieron las condiciones necesarias, la aparición de la vida basada en la química del carbono.

Y finalmente, en lo que a los seres humanos concierne, la aparición de seres inteligentes y la formación de las sociedades.

Esta explicación standar de la evolución del universo conocido hay que completarla con dos nuevos ingredientes que la cosmología actual ha acabado por incluir como necesarios: la existencia de materia y energía oscura de las que se tiene constancia aunque no se ha llegado a detectar experimentalmente, pero que se consideran presentes en el universo de forma que la materia ordinaria representa el $5 \%$, la materia oscura el $27 \%$ y la energía oscura el $68 \%$ (energía y materia son equivalentes teniendo en cuenta la conocida relación de Einstein $\mathrm{E}=\mathrm{mc}^{2}$ ).

Una de las características más destacadas de esta evolución seria la heterogeneidad de las distintas fases, por lo que tiene interés encontrar aquellos aspectos que permitan hilarlas como una evolución con elementos unitarios aunque tengan apariencias distintas en cada una de ellas. Así, en este proceso se pueden observar las siguientes características:

La evolución del universo tiene lugar con una expansión del espacio que conlleva una disminución de la densidad de energía (energía por unidad de volumen) apreciándose en un descenso de la temperatura. Se cree que la temperatura en el momento inicial era de 1032 grados Kelvin $\left({ }^{\circ} \mathrm{K}\right)$ y que en el final del universo será de menos $273^{\circ} \mathrm{K}$ (cero absoluto). Esta transformación se ha analizado desde el punto de vista de la termodinámica y supone el paso de un estado de mínima entropía y máxima energía $\left(1032^{\circ} \mathrm{K}\right)$, representado por una fluctuación o pulso cuántico que se podría considerar el universo en su sencillez y orden 
Miguel Ángel Ortega Sánchez

máximo, a un estado de máximo desorden y mínima energía $\left(-273^{\circ} \mathrm{K}\right)$. La flecha del tiempo $\left({ }^{6}\right)$ marea el paso entre estos dos estados, caracterizado por el principio de uso eficiente de la transformación de la energía que conllevan todos los procesos y que se conoce como «principios de mínimo»: todo suceso que tiene lugar entre dos estados sucesivos se lleva a cabo con un consumo mínimo de energía.

El universo se organiza en base a entidades individuales: partículas (fotones, Quarks...) en el universo de Plank; elementos básicos como el hidrógeno y el helio... en el universo opaco; estrellas, galaxias, ... en el universo oscuro; planetas, sistemas solares... finalmente unidades celulares que se replican y organismos complejos con estructuras orgánicas especializadas. De forma que la individualización parece el mecanismo para la formación de estructuras complejas en la organización del universo.

En la última fase, sobresale la formación de seres vivos individuales y diferenciados cuya evolución conduce a la constitución de organismos complejos sobre la base de subsistemas que determinan el hecho de la vida, en algunos casos como seres conscientes. Su síntesis esta prefigurada en el $\mathrm{ADN}$ de la especie que se va configurando con su relación permanente con el medio dando lugar a un proceso evolutivo que supera el determinismo original que marcaría el estricto desarrollo del programa contenido en su ADN. En este sentido es interesante destacar que cada uno de los subsistemas desempeña una serie de funciones de cierta complejidad, pese a ello la formación de este tipo de seres vivos se ha llevado en un espacio de tiempo que se puede calificar de corto dando la impresión de que los elementos, los procesos, las estructuras, etc. desarrollados por la naturaleza están orientados para su creación. En este sentido, compárese el tiempo que llevan los procesos geológicos que son mucho más simples pero han requerido mucho más tiempo para la formación de ciertas estructuras que no tienen la sofisticación de los seres vivos.

Finalmente nos encontramos en el estadio último de la evolución con la formación de grupos de individuos que se da tanto en el reino animal como en los seres humanos y que constituyen estructuras sociales tipo. Sin embargo las organizaciones humanas han alcanzado

6. Blanco Laserna, D. "La Flecha del Tiempo", en RBA Ediciones. 2015. 
una complejidad que no tiene parangón con los grupos de otras especies. No solamente por su estructura en los distintos ámbitos económicos, jurídicos y sociales sino por los aspectos culturales y científico-téenicos desarrollados.

Por lo tanto, al final de la secuencia se produce el hecho, sorprendente, de la aparición de la inteligencia y la consciencia que permite el reconocimiento de los hechos más destacados siendo posible encontrar un sentido a dicha secuencia de acontecimientos. Finalmente, su valoración orientaría las decisiones en la dirección marcada por el sentido de su evolución.

Esta visión supone la superación de la idea de una existencia eterna e invariable en un universo infinito y eterno y nos conduce al resultado definitivo de una existencia y un universo limitados en el tiempo y en el espacio con un principio y un fin que hoy en día se puede aventurar a la luz de los conocimientos científicos. Este sentido direccional y teleológico debería plantearnos la cuestión del posible sentido del universo y su evolución más allá de que su existencia fuera el resultado de un hecho fortuito del azar, altamente improbable por otro lado, y que por lo tanto le despojara de todo sentido.

Entre otros aspectos que se pueden conjeturar de esta evolución de acuerdo con lo dicho en las líneas anteriores se podrían destacar:

La formación del espacio-tiempo surgido de un pulso inicial de un campo cuántico, muestra una dirección en su evolución marcada por la flecha del tiempo, de un estado de máxima energía y mínimo desorden a otro de mínima energía y máximo desorden.

Como resultado de ese salto de energía del pulso inicial, que representa la fluctuación cuántica, tiene lugar una gran vibración produciendo ondas de distintas longitud de onda y frecuencia en base a saltos cuánticos apareciendo nuevas vibraciones conforme aumentaba de forma continua el espacio con el paso del tiempo, dando lugar a ondas de mayor longitud y menor frecuencia de acuerdo con la relación $\lambda . v=\mathrm{c}(\lambda$, longitud de onda; $v$, frecuencia). Entre salto y salto cuántico las ondas existentes se multiplican exponencialmente en número, al reflejarse en el los "contornos interiores" establecidos por la energía oscura que de acuerdo con la física actual mantiene en el tiempo una cantidad permanente de energía por unidad de volumen (esta conjetura parte de la idea de que el universo no tiene contornos 
Miguel Ángel Ortega Sánchez

como no los tiene la superficie de una esfera, de forma que las reflexiones de los campos cuánticos vibratorios vendrían de su interior como las ondas de un líquido en la superficie de una esfera que chocan contra islas móviles —energía oscura-que flotan en el mismo).

Dado que no se puede producir nada en la nada (Parménides: la nada no es, por tanto no existe), hay que plantear la hipótesis de la existencia de una sustancia base última o primigenia sobre la que cabe conjeturar ciertas propiedades como la de ser infinitamente continua, o de otra forma, tener la «potencia del continuo», esto permitiría su estudio bajo consideraciones de tipo topológico quedando el tiempoespacio ordinario, tal y como muestra la teoría de la relatividad, como una representación de los sentidos a escala humana que habría que considerar cuántico-numerable.

El hecho de que las vibraciones de alta frecuencia de los primeros instantes del universo se condensen en trenes de onda que impidan el principio de superposición ha implicado que se hable de partículas materiales, que interactúan mediante distintos campos cuánticos cuyo origen es la creación de partículas en el "mar de Dirac" o lo que sería los mismo, la aparición de vibraciones en el... "vacio cuántico". En este sentido es clásica la idea de dualidad onda-partícula de forma que a toda onda se le puede asociar una partícula como por ejemplo a las ondas de gravedad, los gravitones, que recientemente se han observado experimentalmente. En realidad, pese a la analogía material de las partículas parece más natural su carácter ondulatorio.

De esta forma se justifica que el universo tenga una naturaleza de carácter ondulatorio que vibra en una sustancia básica. En $\left({ }^{7}\right)$ Robert Botet y Marek Pioszajczak explican el mundo macroscópico a escala humana por medio del Teorema Central de Límite de forma que procesos aleatorios a escala cuántica por la ley de los grandes números se convierten en procesos gobernados por leyes estables que explican los cambios y transformaciones del mudo físico.

La existencia de leyes que permiten predecir la evolución de los sistemas físicos no presupone la existencia de un determinismo. Es decir, hay tendencias que determinan la evolución, pero se pueden producir

7. Robert Botet, Marek Pioszajczak: Universal fluctuations. The Phenomenology of Hadronic Matter. World Scientific Lecture Notes in Physics Vol. 65. 2002. 
efectos locales que van en sentido contrario: la expansión en el universo es la norma general pero localmente se pueden producir acercamientos aleatorios. Gráficamente, de la misma forma que en un rio la tendencia general del movimiento del agua viene determinada por una diferencia de alturas entre el inicio y final de la corriente, localmente se pueden producir turbulencias que vayan en sentido contrario a la corriente debidas a procesos de inestabilidades locales impredecibles.

Otra característica de la evolución es la diversidad de formas que ha tomando esa evolución en las distintas fases de la expansión: formación de partículas elementales y átomos de elementos simples, aparición de estrellas en grandes nubes de gas y finalmente la formación de elementos pesados en el núcleo de las estrellas que tras un proceso de descomposición estelar pasan a sistemas planetarios en los que bajo ciertas condiciones los elementos del carbono dan lugar a la química orgánica que permite la aparición de macromoléculas de las que surgen las propiedades de la vida.

El sentido, si existe, de los hechos que aparecen en esa secuencia está por establecer. Por ahora no se puede explicar el sentido del universo ni siquiera desde el punto de vista físico aunque se puede aventurar el inicio de todo el universo en una fluctuación de un campo cuántico (Big Bang) y su final de máxima entropía con una uniformidad tal que no habrá puntos entre los que haya diferencia de estados de energía. Pero tampoco desde el orden lógico, estético, ético, moral o intelectual. El sentido social y humano de la realidad desde estos puntos de vista sería subjetivo. Bertrand Russell, con el típico escepticismo británico, lo expresó diciendo:

Puede que el universo tenga un propósito, pero no se conoce nada que sugiera que, de ser así, ese propósito tenga algo que ver con el nuestro.

No obstante, lo anterior, sí es posible encontrar algún principio que explique la evolución de los sistemas físicos y sociales y derivar, de este principio, sus implicaciones sociales. La física clásica y en particular una de sus ramas la «Mecánica Racional» que explica el estado de movimiento o reposo de los cuerpos, utiliza lo que se denomina 
«principios de mínimo» que afirma, como se ha indicado anteriormente, que los estados que adopta la naturaleza se producen mediante la transformación o consumo de un mínimo de energía y si el proceso es dinámico, a través de una serie de estados que evolucionan a lo largo del tiempo, de forma que, cada uno de ellos y la transformación que representa su evolución supone un mínimo de energía. Así lo recogió Pierre-Louis Moreau de Maupertuis (1744) (ver los comentarios del trabajo de Vicente Menéndez $\left({ }^{8}\right)$ ) y así lo expresaron en sus ecuaciones: Lagrange, Hamilton, y finalmente Hamilton-Jacobi.

Estos principios tenían sus equivalentes en otras ramas de la física como la óptica (Principio de Fermat) que dice que el camino recorrido por la luz entre dos puntos tiene la mínima longitud posible.

En el campo de las ciencias sociales y en particular en la economía, con el planteamiento de la escuela neoclásica o marginalista, las decisiones de los agentes económicos se justificaban considerando que estos optimizaban su posición (máximos ingresos, mínimos costes en las empresas, máxima utilidad en los individuos, minimización de gastos, etc.). Como resultado de esta optimización, el agregado de todos los agentes económicos supone un óptimo que se conoce como «Óptimo Paretiano» que se suele expresar diciendo que es la situación de equilibrio que modificada alguien sale perjudicado.

La optimización, por tanto, en el caso de la actividad económica viene a representar el uso eficiente de los recursos (hacer lo máximo con lo mínimo), lo que supone una optimización desde el punto de vista físico, punto en el que se une de alguna manera el entorno físico y el medio social.

Así mismo tanto en física como en economía los principios de óptimo representan, como se ha indicado para el Óptimo Paretiano situaciones de equilibrio estático o dinámico de la misma forma que

9. Vicente Menéndez, V. "El principio de mínima acción de Maupertuis: el sueño de una visión unificadora del mundo en el siǵlo XVIII, o la búsqueda de Dios a través de la belleza y simplicidad de las teorías", en Facultad de Ciencias Exactas y Naturales. Universidad de Buenos Aires. 
las ecuaciones de óptimo de Hamilton-Jacobi encierran las condiciones de equilibrio dinámico (ecuaciones de Newton). Su aplicación al campo económico puede verse en $\left(^{9}\right)$.

Además, el concepto de equilibrio permite la analogía entre el campo físico y el social. Las moléculas de un gas contenido en un volumen dado están en constante interaceión buscando una posición de equilibrio que suponga la mínima energía. Si existiera esa posición las moléculas se moverían hasta ocuparla y permanecer en ella de forma estable. El hecho de la agiitación de las moléculas en el volumen parece indicar que las distintas instantáneas de la agiitación representan mínimos de energía o posiciones próximas al entorno mínimo. Desde el punto de vista termodinámico estos estados representan en general, la máxima entropía. De forma análoga en un sistema económico o social en unas condiciones dadas en el que todos los agentes económicos o sociales interactúan unos con otros, se llega a un estado de equilibrio entorno a una posición de óptimo en la que se ha producido una homogeneización de las magnitudes que definen el equilibrio. Esta uniformización se podría considerar también como una situación de máxima entropía.

Por tanto, la idea de optimización y también la definición de estados de equilibrio, por analogía se puede decir que son equivalentes o semejantes en el ámbito físico y económico: mínima energía requerida para producir un cambio entre dos estados físicos y mínimo gasto de recursos para alcanzar determinados objetivos económicos. Por lo que a los principios de óptimo se les puede atribuir un carácter universal, pudiéndose decir que son principios que rigen con carácter general pero de los cuales no se puede inferir un sentido. Más adelante se verá como la idea de optimización que conllevan los principios de mínimo se puede extender a los procesos de carácter social.

10. Fernández Díaz, A. (director): "Fundamentos y papel actual de la política económica". Editorial Pirámide. 1999. 
Miguel Ángel Ortega Sánchez

\section{El universo inteligente y consciente}

Pese a las previsibles condiciones de inestabilidad que condicionan el problema dinámico de los primeros momentos, ha surgido un universo estable que evoluciona de forma apacible aunque localmente pueden darse sucesos inestables. Es probable que las leyes y ecuaciones que modelizan la formación del universo sean de carácter fuertemente no lineal y que por lo tanto impliquen que variaciones de partida y a lo largo del proceso, den lugar a estados imprevisibles dentro del carácter caótico que caracteriza este tipo de fenómenos. Pese a ello se crean condiciones para la aparición de seres vivos y más sorprendentemente de seres vivos con inteligencia y capacidad de consciencia. En este sentido es famosa la cita de Kant:

«Dos cosas llenan mi ánimo de creciente admiración y respeto a medida que pienso y profundizo en ellas: el cielo estrellado sobre mi y la ley moral dentro de mi»

A lo largo de la segunda mitad del siglo XX estos planteamientos han tomado forma en la idea del Principio Antrópico. Así, «El biólogo John Burdon Sanderson Haldane apuntó hacia una posible relación entre la estructura del universo como un todo y las condiciones locales necesarias para que surja la vida. Tal vez la bioquímica en la que se basa la vida, decía Haldane, solo haya sido posible en una época específica de la evolución del universo.

En relación con el origen de la vida, a finales de la década de 1950, el astrofísico estadounidense Robert Dike expuso el siguiente razonamiento. Una condición necesaria para la aparición de cualquier tipo de vida es que existan átomos más pesado que el helio, como el carbono o el oxígeno. En el Big Bang solo se formaron átomos de hidrógeno y de helio, con algunas trazas de deuterio o litio. Los átomos más pesados se forman posteriormente en las reacciones nucleares que tienen lugar en el interior de las estrellas. En una serie de etapas sucesivas, el hidrogeno inicial se transforma en helio, este en carbono y así hasta llegar al hierro-níquel. En este punto, si la masa de la estrella es suficientemente grande, se produce una explosiónimplosión, emitiendo al espacio los átomos que contienen, formando una estrella de neutrones y finalmente un agujero negro. Por lo tanto, para que exista la vida es necesario que se hayan formado estrellas, 
que algunas hayan muerto después de haber producido y difundido átomos pesados y que existan estrellas como el Sol, en fase de combustión de hidrogeno. Todo esto requiere un tiempo de $10^{10}$ años. Siguiendo razonamientos físicos Dike piensa que la edad del universo está condicionada por factores biológicos. El astrofísico australiano Brandon Carter llegó independientemente a conclusiones parecidas, pero las situó en un contexto más general que llamó Principio Antrópico. Con su principio Carter quería subrayar que la presencia de la vida es un acontecimiento muy raro que no tiene nada de inevitable, sino que depende de factores aleatorios. Otros científicos como John Barrow y Frank Tipler fueron más allá y enunciaron el Principio Antrópico fuerte, frente al débil de Carter, que más o menos viene a decir que el universo implica necesariamente la presencia de vida inteligente. Su existencia resulta inevitable por un ajuste muy fino de los valores de las constantes universales» $\left({ }^{10}\right)$.

Por tanto, eabe la posibilidad de que el sentido del universo físico fuera la creación orientada a la formación de las condiciones para la existencia de la vida inteligente. Los resultados de este hecho también deberían ser analizados:

El primer resultado de la aparición de la inteligencia seria la posibilidad del autoconocimiento del propio universo que ha dado lugar a esa inteligencia.

Otra, como ya se ha indicado, es la formación de una conciencia moral.

Si el universo en su evolución ha llegado a formación de entidades con una conciencia ética sobre lo que es racional, lógico, bueno, justo y bello, ... y también sobre lo que no lo es, quizás esta evolución tiene algún sentido tanto en el camino seguido, los procedimientos empleados y los fines que persigue.

Pues como señala Kant:

...un producto organizado de la naturaleza es aquel en el cual todo es fin y recíprocamente, también medio. Nada en él es en balde, sin fin atribuible a un ciego mecanismo natural.

11. Blanco Laserna, D. "La Flecha del Tiempo", en RBA Ediciones. 2015. 
Además, como el funcionamiento de nuestras facultades está conforme a la naturaleza, la causa de nuestras facultades y de la naturaleza tenía que ser la misma. La pregunta siguiente sería, de manera natural, qué fin debía perseguir esta causa del mundo. No podía ser ningún fin relativo o condicionado, sino un fin incondicionado. Ahora bien, fin incondicionado o fin en sí mismo es solo el hombre como ser moral. Si la naturaleza había sido creada para el hombre como ser moral es que estaba pensada para adaptarse a los fines de la libertad. Entonces, la consideración finalista de la naturaleza era como la condición de posibilidad de la acción moral en el mundo. En suma, esa exigencia de la razón que Kant llamaba "la finalidad de la naturaleza", cuya expresión era el juicio teleológico, constituía el nexo entre lo sensible y lo inteligible y capacitaba para pensar la naturaleza como un espacio donde era posible la realización de los fines en harmonía con las leyes naturales.» $\left({ }^{11}\right)$.

El hecho extraordinario de la existencia de vida inteligente organizada socialmente es singular de nuestro planeta y desconocido fuera del mismo, aunque el Principio Antrópico, dado el número de galaxias, estrellas así como de sistemas planetarios y admitiendo las condiciones generales de homogeneidad del universo, apunta la posibilidad de que se esté produciendo en otros lugares del universo.

Analizar, por tanto, el sentido de la formación y desarrollo de vida inteligente nos limita a partir de aquí a la historia social de la especie humana. En este sentido se van a recoger de forma resumida los planteamientos teóricos desde el punto de vista institucional (ya que este constituye la referencia de este trabajo), tomando las aportaciones de autores clásicos y partiendo de la dialéctica que resulta de la dualidad individuo sociedad todo ello situado en un mareo evolucionista.

Así, históricamente llama la atención la formación de grandes organizaciones sociales que han conseguido una compleja estructura social que se fundamenta en la formulación de una concepción de la existencia humana. Sociedades que durante un período de tiempo

12. Francisco Manuel Arroyo García, F. M.; Marcos Jaén, M. "Kant ¿Qué podemos saber y qué podemos hacer? En busca de los límites del conocimiento y de la moral”, en RBA, 2015.

Naturaleza y Libertad. Número 11, 2019. ISSN: 2254-9668 
muestran un impulso expansivo que se extienden imponiendo su sistema a los grupos sociales que caen bajo su influencia. Son los grandes grupos de sociedades conocidos como Imperios y Civilizaciones.

Como indica Guy Rocher en su Introducción a la Sociología General $\left({ }^{12}\right)$, "Tres problemas principales presiden la indagación teórica y empírica en la sociología. Cabe enunciarlos en los términos siguientes:

- ¿Cómo explicar la existencia y permanencia de las colectividades humanas? y , correlativamente ¿cómo explicar la inserción del individuo en esas colectividades?

—¿Cómo se organizan o estructuran los mareos sociales de la vida humana?

—¿Cómo se produce y se explica el cambio, la evolución de las sociedades humanas?

El primer interrogante plantea el problema de la acción social, el segundo el de la organización social y el tercero, el del cambio social y el de la acción histórica".

En el análisis de la evolución de las sociedades hay que busear los elementos que las caracterizan. El hecho más elemental, pero esencial de la sociedad es que está formado por individuos que adoptan una forma de organización y un sistema de relaciones. La creciente complejidad de la evolución de las sociedades pone de manifiesto aspectos de diferente naturaleza como los aspectos sociales, económicos, políticos, jurídicos... en todos ellos se encuentra su esencia institucional. En el sentido que aquí se quiere dar a la idea de institución, uno de los primeros autores que hace referencia a esta idea fue Maurice Hauriou (1856-1929), «quien expresó la problemática de la estructura de la sociedad en una sugestiva y simple teoría: la estructura de la sociedad es dualista, en parte objetiva y en parte subjetiva. Si se la compara con un tejido, dice con bellísima metáfora, se puede decir que la urdimbre de este tejido representa el elemento del orden y está formado por ideas objetivas mientras que la trama, que representa los elementos del poder y la libertad, está formada por la voluntad y las pasiones subjetivas de los hombres. El contenido de esa sociedad ofrece dos objetos de conocimiento: los acontecimientos que son la obra subjetiva

13. Guy Rocher: "Introducción a la Sociología General”. Editorial Herder. 1973. 
de los hombres, y las instituciones, que son el sedimento objetivo. Estas instituciones son todo elemento de la sociedad cuya duración no depende de la voluntad de los individuos determinados» $\left({ }^{13}\right)$.

Houriou estableció de esta forma la distinción entre aquellas relaciones reciprocas de los individuos que Max Weber definió como conductas humanas referidas a otro y que constituyen el sentido subjetivo de la sociedad frente a las formas de obrar, pensar y sentir que se imponen como una presión anónima y que expresan símbolos, normas de acción y sentimientos que se dan en un conjunto de individuos que apareeen unidos entre sí como un grupo y que Ortega y Gasset denominó como la consistencia anónima de las manifestaciones objetivas de la vida social.

Se atribuye a los trabajos de Thornstein B. Veblen (1857-1929) $\left({ }^{14}\right)$ el nacimiento de la corriente institucionalista que en épocas más recientes se ha desarrollado en múltiples direcciones como las de James Buchanan, Ronald Coase, Douglas North, etc. entre otros. La idea de institución para los distintos autores puede tener distintas interpretaciones, pero en cualquier caso, tienen un carácter más restringido que el de M. Hauriou, ya que en general se refieren a organizaciones concretas del ámbito económico y político. Mientras que el concepto de Hauriou hace referencia, además, a las pautas de los comportamientos individuales, a las formas culturales, los hábitos sociales, etc., como se recoge en los trabajos de Deirdre N. McCloskey $\left[\left({ }^{15}\right),\left({ }^{16}\right),\left({ }^{17}\right)\right]$.

La mayor parte de los autores desde Augusto Compte, Herbert Spencer, Emile Durkheim ponen de manifiesto la complejidad organizativa de la sociedad industrial (Comte), la creciente heterogeneidad (Spencer) o la diversidad (Durkheim) y la diferenciación que afecta

14. Sánchez Agesta, L. "Principios de Teoría Política”. Sexta edición, en Editora Nacional, 1979.

15. Veblen, T. B. "Teoría de la clase ociosa". Fondo de Cultura Económica.1971.

16. McCloskey, Deirdre N. "The Bourgeois Virtues Ethics for an Age of Commerce", en The University of Chicago Press. 2006.

17. McCloskey, Deirdre N. "Bourgeois Dignity Why Economies Can't Explain the Modern World", The University of Chicago Press. 2008.

18. McCloskey, Deirdre N. McCloskey, Deirdre N. "Bourgeois Equality", en The University of Chicago Press, 2010. 
tanto a la organización y su estructura como a las formas de comportamiento y la cultura que requiere como contrapartida el desarrollo de procesos de integración, cooperación espontánea (Spencer) o de complementariedad y la solidaridad orgánica (Durkheim). Precisamente Emile Durkheim acertó al prever el creciente individualismo que defendía Spencer junto con la presencia del Estado basado en una burocracia organizativa de Compte. Para Durkheim "el lugar del individuo es mayor y el poder gubernamental menos absoluto. Pero no se da contradicción alguna en el hecho de que la esfera la acción individual se amplíe al mismo tiempo que la del Estado" $\left({ }^{18}\right)$

Ante la creciente complejidad de las sociedades industriales y las dificultades analíticas que conlleva su estudio, a mediados del siglo XX Talcott Parson recupera la teoría institucional $\left({ }^{19}\right)$ situándola dentro de la Teoría General de Sistema de Ludwig Von Bertalanffy $\left({ }^{20}\right)$ con el fin de poder considerar la mayor complejidad posible alcanzada por las sociedades modernas, situándose en una corriente en lo que se ha conocido como neoevolucionista. Parson distingue dos subsistemas: por una parte el subsistema cultural que comprende los valores, los conocimientos, las ideologías, ... es decir el conjunto del aparato simbólico en que se inspira toda la acción social y por otra el subsistema social que concierne a las condiciones implicadas en la interacción de los individuos que forman parte de colectividades coneretas $\left({ }^{21}\right)$. El concepto clave que establece la conjunción y la distinción entre ambos subsistemas es la institucionalización, que consiste en la concreción de los elementos culturales (valores, ideas, símbolos que poseen por naturaleza un carácter general), en unas normas de acción, en unos roles, en unos grupos que ejercen un control directo e inmediato sobre la acción social y la interacción de los individuos del grupo social, planteamientos que recuerdan los iniciales de Hauriou. En su elaborado y teórico modelo, sin embargo, hay que indicar que Parson rechaza la

19. Emil Durkheim. "La división del trabajo social". Prometeo.

20. Leon H. Mayhew (editor): "Talcott Parsonson Institutions and Social Evolution" (Selected Writings). The University of Chicago Press. 1982.

21. Ludwig Von Bertalanffy. "Teoría General de los Sistemas". Fondo de Cultura Económica. 1976.

22. Parson, Talcott: "Theories of Society”. The Free Press of Glencoe, Inc.1961. 
idea de la existencia del determinismo en los individuos en cuanto actores de la vida social.

Recopilando lo anterior, aunque a lo largo de la historia algunas instituciones hayan surgido espontáneamente otras han sido creadas atendiendo a las circunstancias históricas de cada momento de forma que su evolución y permanencia depende de su eficiencia en la obtención de determinados fines. Por ello es posible hablar de un proceso de selección natural de instituciones que tendrá lugar en períodos más o menos largos de tiempo fuera de la influencia de las decisiones de los individuos que las crearon. En este sentido tienen un carácter histórico y va a ser el paso del tiempo el que determine su vigencia. Lo que nos lleva a la conclusión, que crea cierta polémica, de que existe un darwinismo en el ámbito de las instituciones como lo existe en la propia naturaleza en muchas de sus vertientes así como en el ámbito social. Sin embargo no tiene que ser un problema ya que las instituciones que se mantienen hacen al sistema social más eficiente y por tanto estará en mejores condiciones para corregir situaciones anómalas que siempre aparecen en los sistemas naturales porque no hay nada perfecto. Aquí convendría recordar a Ludwig Boltzmann, uno de los físicos más importantes del siǵlo XIX que definió el concepto de entropía de forma rigurosa y fue firme defensor de la teoría atómica de la materia frente a los energetistas, y que era un ferviente seguidor de la Teoría de la Evolución de Darwin.

Como se ve, la acción social se desenvuelve a través del entramado institucional. De forma que el estado de cada momento viene a manifestar la opción social optima que quizá no sea la mejor que se pudiera dar, pero si puede suceder que sea la menos mala entre las posibles. Esto se puede denominar Mecánica Histórica en la que se materializa el óptimo social.

Esta visión es más general que el materialismo histórico el cual viene determinado por los instrumentos de producción que condicionan la vida social, como manifestó Carlos Marx. Por otra parte, la idea de conflicto que plantea Marx bajo la visión del mecanismo Hegeliano Tesis-Antítesis-Síntesis que presuponen los intereses de clase o individuos se ve superada por la idea de acción humana que se desarrolla por medio de las instituciones sociales. 
El conflicto de intereses se podría considerar como una solución equivocada del problema social que causaría su frustración a través de instituciones sociales mal planteadas que no dan salida a la acción de los individuos. Históricamente la superación de los conflictos y la frustración social vendría acompañada de cambios en la organización social, en las costumbres, etc. en definitiva por nuevas instituciones $\left({ }^{22}\right)$.

Desde el punto de vista de la dualidad individuo-sociedad, la vertiente individual parece que puede justificarse con el motivo de facilitar una mayor diversidad de posibles variantes de acción, ya que cada individuo podría representar una opción diferente de forma que la contraposición de opciones permitiría un proceso de selección de las mejores. Por tanto, cabe pensar que la individualidad es necesaria para la formulación de instituciones y por tanto un medio para facilitar la selección así como su adaptación durante la evolución. Desde la vertiente social, como resultado de la suma de acciones humanas se producen unos resultados que van más allá de la dimensión individual. Entre aquellos aspectos que transcienden a la acción humana destacan en particular la institucionalización del desarrollo técnico y el conocimiento científico en todas las vertientes del ser humano desde el mundo físico al social así como su continua difusión a todo el grupo social. Este desarrollo científico-técnico se debe de manera particular a la evolución de la organización social que busca la capacidad de adaptación basada en la diversidad lo cual supone la creciente complejidad social y el aumento de las interrelaciones entre los individuos que el desarrollo tecnológico favorece.

Enlazando con la introducción, se puede considerar que el permanente y continuo progreso del conocimiento afecta a la propia comprensión y concepción que el hombre tiene de sí mismo, de las instituciones y de los principios básicos que informan la naturaleza de las mismas. Así es posible que con el paso del tiempo se llegue a modificar el sentido de algunos conceptos como la justicia, la libertad, igualdad, etc. que forman parte del carácter social de la naturaleza humana de manera que si continua el proceso de regulación, organización y control previsto por Compte y Durkheim paralelo a la 
Miguel Ángel Ortega Sánchez

creciente complejidad de las sociedades y sus instituciones, será necesario conocer las categorías conceptuales que caracterizan la naturaleza de las mismas y establecer los principios generales que permitan encontrar unos criterios simples y claros de regulación que al mismo tiempo hagan posible una organización eficiente y faciliten su capacidad de adaptación.

Un mejor conocimiento de la historia de la evolución social y de la secuencia de instituciones que va dejando tras de sí, ayudaría a encontrar las claves de dicha evolución y al mismo tiempo comprender su relación con la naturaleza y su sentido en un universo en el que las leyes físicas que lo configuran parecen estar guiadas por el Principio Antrópico que conduce al desarrollo de seres inteligentes con conciencia moral la cual determina la orientación de sus decisiones dirigidas hacia un fin que por tanto marcaria el sentido de su existencia.

\section{Conclusiones}

En el presente trabajo se ha pretendido sintetizar la evolución del universo desde su origen hasta la formación de sociedades humanas en las que finalmente se ha desarrollado la inteligencia, la consciencia y la conciencia moral con el fin de tener una visión global y justificar una «teoría del Todo más allá de la Física» que permita contemplar la existencia humana tanto en su vertiente físico-natural y social como algo único. Se ha empezado tratando de encontrar la unificación de las leyes físicas en una idea simple que exprese la naturaleza del universo llegándose a la conclusión que el universo se puede considerar de forma gráfica, como una gran vibración de un campo cuántico en la que adquieren presencia y se manifiestan tanto las "partículas" elementales componentes de la materia como las fuerzas básicas de la naturaleza (fuerzas nucleares fuerte y débil, gravitatoria, ...) que hoy se trata de formular matemáticamente mediante la teoría (sin confirmación experimental) de las supercuerdas y la supersimetría (Teoría SUSY) y que de alguna manera debería recogerse en una ecuación de ondas que particularizada en las condiciones de cada momento explicaría la imagen del universo (un ejemplo de esto es la ecuación de ondas de Schrödinger), mareada por una profunda asimetría que señala la flecha del tiempo, produciéndose una sucesión de estados 
desde uno inicial, ordenado, de máxima energía a otro final totalmente desordenado y con mínima energía, como la corriente de un rio que desciende de un nivel a otro inferior, en cuya evolución se van a desenvolver los acontecimientos de la historia. Los individuos van a formar parte de esta corriente actuando en sus ámbitos de libertad como las gotas de agua se mueven en la corriente que las arrastra.

En esta visión de la historia del universo se ha descrito el proceso físico que ha llevado a la formación de vida consciente poniéndose de manifiesto la complejidad del proceso teniendo en cuenta las múltiples formas que puede adoptar el universo, sin embargo parece que este ha tomado el camino que lleva a la aparición de la vida consciente como una condición sine equa non de su existencia. Por tanto, el hecho de la presencia de vida inteligente, supuesta la ínfima probabilidad de su aparición lleva a pensar que el universo tiene implícito un mecanismo cuya finalidad es la producción de vida inteligente con criterios lógicos, éticos y morales (Principio Antrópico). Pudiéndose decir que en estas condiciones, el universo ha tomado conciencia de sí mismo. ¿Tiene esto algún sentido en un universo destinado a desaparecer?: está cuestión hoy no tiene respuesta. Pero es probable que con el tiempo el avance de la civilización con su desarrollo científico, intelectual, cultural sea capaz de establecer su sentido.

A partir de la aparición de vida consciente se ha seguido la evolución de las sociedades humanas, en las que destaca su carácter dual: por una parte la tendencia individual de los intereses personales y por otro la necesidad social de colaboración o participación impulsada por ese mismo interés individual. Bajo este impulso los grupos humanos han pasado por distintos tipos de formaciones sociales hasta formar grandes grupos humanos como los Imperios y las Civilizaciones. El análisis de la evolución de los mismos y las características de las sociedades avanzadas ayudan a comprender la naturaleza de los fenómenos sociales. Así a partir de la revolución industrial se pone claramente de manifiesto la creciente diversidad y la diferenciación social provocando una mayor interdependencia favorecida por el continuo desarrollo científico-técnico que conduce a un aumento de la capacidad de adaptación y eficiencia económica. La mayor interrelación y complejidad social supone una multiplicación de las instituciones que 
Miguel Ángel Ortega Sánchez

dan forma al sistema de relaciones sociales creando nuevas condiciones para un desarrollo cultural que afecta a los hábitos de comportamiento y a las conductas morales orientando las decisiones individuales y sociales que marcarían la dirección del cambio y la evolución social.

Desde esta visión conjunta de los fenómenos físicos y de la evolución social se han tratado de buscar los principios que los caracterizan y el sentido de los mismos con el fin de encontrar las pautas que puedan orientar la conducta humana. De acuerdo con este planteamiento se ha justificado como los principios de óptimo, que tienen amplia aceptación en la física en los principios de mínimo, se pueden extender al campo económico con la idea de Óptimo Paretiano. Por otra parte, la vertiente social de la naturaleza humana se ha radiografiado mediante el concepto de institución que recoge la idea de eficacia resultando la translación de la idea de óptimo al campo social con la generalización de la teoría darwinista de la selección.

Por tanto, la condición de eficiencia orienta la acción individual y social mientras que las pautas de conducta marcan la orientación de sus decisiones. Sin embargo es todavía es difícil llegar a establecer un sentido en los cambios y las transformaciones sociales que a su vez den sentido a la existencia humana. Se puede decir que no sabemos lo que tenemos que hacer (sentido) pero si lo supiéramos, sí que sabríamos como hacerlo (con eficiencia). Mientras tanto orientamos nuestras acciones con la luz del conocimiento y la razón, buscando en los descubrimientos científicos el camino que nos lleve al destino final que la naturaleza del universo tiene fijado (si lo tiene), mientras llega ese momento no se puede hacer otra cosa que tratar de buscar la felicidad de los seres humanos en el entorno que les ha tocado vivir.

Miguel Ángel Ortega mon@empre.es 